\title{
Strategies for Numerical Integration of Discontinuous DAE Models
}

\author{
Domingos Fabiano de S. Souza, Roberta C. Vieira and Evaristo C. Biscaia Jr* \\ Programa de Engenharia Química, PEQ/COPPE/UFRJ, Universidade Federal do Rio de \\ Janeiro, CP 68.502, CEP 21.945-970, Rio de Janeiro, Brasil \\ * corresponding author, evaristo@peq.coppe.ufrj.br
}

\begin{abstract}
In this work, it is presented a novel strategy to solve discontinuous DAEs of floating index type. The switching between DAE models of different indexes and the reinitialization of the system are performed automatically by the code. The direct integration of the high index DAE model is aided by the software. The equation actually fed to the numerical integrator is a weighted sum of the different model equations. The weights are either 0 or 1 inside integration intervals, depending on whether the corresponding equation is active or not during the time interval in consideration. Across model discontinuities, the numerical values of the weights are taken from 0 to 1 (or vice-versa) via a smooth regularization function, which is a continuous representation of a step function. Several functions are suitable to perform such task, and the authors suggest a family of functions which are simple and differentiable up to the order needed. As an example, the optimal control of a fedbatch fermentation (production of ethanol by $S$. cerevisiae ) is presented.
\end{abstract}

Keywords: dynamic optimization, floating index DAEs, fedbatch reactors

\section{Introduction}

In many dynamic optimization problems, the differential index of the DAE model changes in time, as a consequence of the activation of problem-specific constraints. This class of systems has been addressed as Floating Index DAE Problems. The index of a DAE is the number of times that all or a part of a DAE must be differentiated with respect to time in order to convert it into an explicit set of first order ordinary differential equations (ODEs). Index 1 systems may be solved with modified ODE methods, while higher index systems (systems with index 2 or greater) require specific methods. Generally, higher index systems are rewritten by means of an index 1 formulation and solved with standard integration codes. Whichever the strategy adopted, reinitialization is required in order to restart integration after the model discontinuity.

Singular optimal control problems, as in the fedbatch operation of chemical reactors, are typical problems. At the start of the operation, the problem consists of a typical optimal control optimization problem, and the control variable is to be set at one of its extreme values. The resulting DAE model presents index 1 . When the singular arc is reached, 
the singular control constraint becomes active, and the index of the DAE model shifts to 3. The singular interval finishes when a physical constraint of the system is reached (typically the maximum volume of the reactor). From that time on, the optimal control problem is represented by an index 1 DAE.

Frequently, discontinuities in the state variables are observed at the model switching point. Hence, the final state of the previous model can not be used as initial condition to start the simulation of the subsequent model, and a reinitialization procedure of some sort must be carried out.

The following steps are needed for the numerical resolution of index one systems with model discontinuities: (i) detection of activation/deactivation of constraints (model discontinuities); (ii) model switching; and (iii) determination of consistent initial conditions to restart integration (Majer et al., 1995, Park and Barton, 1996, Wu and White, 2001). For higher index systems, index determination (and frequently index reduction) are further required (Feherry and Barton, 1998). Several algorithms to perform those tasks have been reported (Vassiliadis et al., 1994a e 1994b; Feehery and Barton, 1996, 1998; Asher and Petzold, 1998), and the high computational effort typically associated with each of these steps motivated the present work, which aims the reduction of the effort associated with the numerical resolution of floating index DAEs.

\section{Resolution of Floating Index DAEs}

Consider the general DAE system consisting of Eq.1 and Eq.2a when the constraint is inactive and Eq.1 and Eq.2b when the constraint is inactive. This system has been addressed by Feehery and Barton (1998) as a disjunctive system, based on the fact Eq. $2 \mathrm{a}$ and $2 \mathrm{~b}$ can not be active at the same time.

$$
\begin{aligned}
& \mathrm{f}(\dot{\mathrm{x}}, \mathrm{x}, \mathrm{y}, \mathrm{u})=0 \\
& \mathrm{u}-\mathrm{u}^{\mathrm{c}}=0 \\
& \mathrm{~g}(\mathrm{x}, \mathrm{y})=0
\end{aligned}
$$

In the present work, a novel methodology for its numerical resolution is presented. The disjunctive part of the system (Eq. $2 \mathrm{a}$ and $2 \mathrm{~b}$ ) is replaced by a single algebraic equation, which represents the weighted sum of the excluding equations.

$$
\eta\left(u-u^{c}\right)+(1-\eta) \cdot g(x, y)=0
$$

The weight $\eta$ is set to 1 when the constraint is inactive, to 0 when it is active and to an intermediate value in the interval $[0,1]$ during the switching period. Due to this smooth transition from one system to the other, the state variables are continuous along the model switching and no reinitialization algorithm is required to continue integration.

The utilization of regularization functions to circumvent initialization problems has been studied by Vieira and Biscaia Jr. (2000). The authors have established some criteria to guide the selection of a regularization function and its parameters. The function chosen for he present work is shown in Eq. 4, where $\Delta \mathrm{t}$ represents the time elapsed since the model switching. Parameters $\tau$ and $n$ are to be set by the user. The regularization function is differentiable up to order (n-1), and it approaches a true step 
function as the $\tau$ parameter gets smaller. Figure 1 shows the impact of both parameters on the regularization function selected.

$$
\eta(\Delta t, n, \tau)=\left\{\begin{array}{cc}
0 & \Delta t<0 \\
1-\exp \left(-\mathrm{n} \frac{\Delta \mathrm{t}}{\tau}\right) \cdot \sum_{\mathrm{j}=0}^{\mathrm{n}-1} \frac{1}{\mathrm{j} !}\left(\mathrm{n} \frac{\Delta \mathrm{t}}{\tau}\right)^{\mathrm{j}} & \Delta \mathrm{t} \geq 0
\end{array}\right.
$$
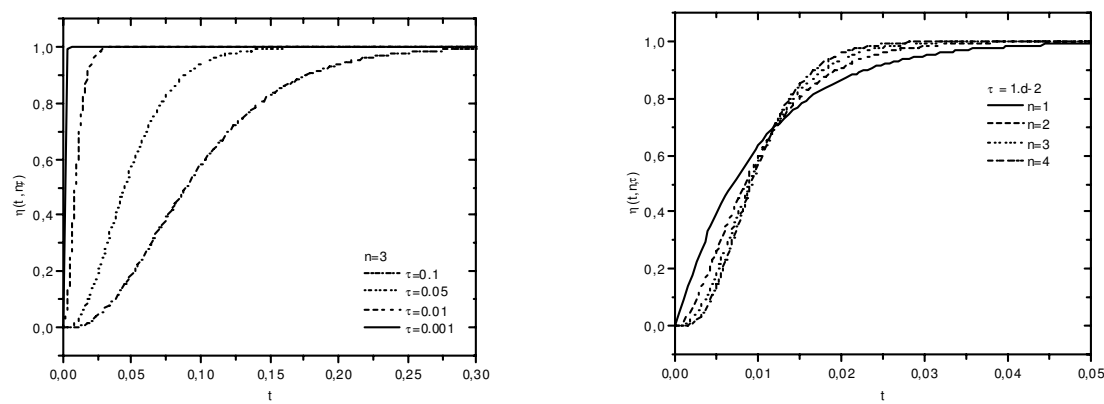

Figure 1 - Impact of parameters on the regularization function selected.

The code PSIDE (Lioen et al., 1998) is used to perform the numerical integration of the DAEs. The code can deal with fully implicit DAE systems of index up to 3 , and its selection eliminates the need of index reduction.

\section{Numerical Example}

In order to show the potentiality of the proposed approach, the problem of maximization of ethanol production presented by Hong (1986) is revisited. The DAE system is comprised by Eq. 5 to 7 :

$\frac{\mathrm{dx}_{1}}{\mathrm{dt}}=\mu \mathrm{x}_{1}$

$\frac{\mathrm{dx}_{2}}{\mathrm{dt}}=\varepsilon \mathrm{x}_{1}$

$\frac{\mathrm{dx}_{3}}{\mathrm{dt}}=\mathrm{u}$

where $\mathrm{x}_{1}, \mathrm{x}_{2}$ and $\mathrm{x}_{3}$ represent the mass of cells, the mass of product (ethanol) and the volume of the system, respectively. The substrate addition rate $\mathrm{u}$ is the manipulated variable, and the goal is to maximize the amount of ethanol at the end of the batch $\left(\mathrm{t}=\mathrm{t}_{\mathrm{f}}\right)$.

The growth rate, $\mu$, and the specific production, $\varepsilon$, are functions of $S$ (substrate concentration) and $\mathrm{P}$ (product concentration), where $\mathrm{S}=\mathrm{S}_{\mathrm{f}}+\left(\mathrm{c}-\mathrm{x}_{1}\right) /\left(0.1 \mathrm{x}_{3}\right)$ and $\mathrm{P}=\mathrm{x}_{2} / \mathrm{x}_{3}$. 
$\mu(S, P)=\frac{\mu_{0} S}{\left(1+\frac{P}{K_{1 p}}\right)\left(K_{1 s}+S\right)}$

$\varepsilon(\mathrm{S}, \mathrm{P})=\frac{\mathrm{g}_{0} \mathrm{~S}}{\left(1+\frac{\mathrm{P}}{\mathrm{K}_{2 \mathrm{p}}}\right)\left(\mathrm{K}_{2 \mathrm{~s}}+\mathrm{S}\right)}$

The optimal control policy can be determined via the Pontryagin Maximum Principle (Pontryagin et al., 1963), being the Hamiltonian H represented as follows:

$\mathrm{H}=\mathrm{H}_{0}+\phi \mathrm{u}=\left(\lambda_{1} \mu+\lambda_{2} \varepsilon\right) \mathrm{x}_{1}+\lambda_{3} \mathrm{u}$

The singular arc condition, $\phi=0$, leads to the elimination of the adjoint variable $\lambda_{3}$ of the system. When the singular arc constraint is active, the control action $u$ that leads to the optimal trajectory is the one that maximizes the Hamiltonian. For this problem, it leads to:

$\lambda_{1} \mu+\lambda_{2} \varepsilon=0$

As $\phi(t)$ and all its derivatives are linear functions on $\lambda$, the differentiation of Eq. 11 generates:

$\lambda_{1} \frac{\partial \mu}{\partial x_{3}}+\lambda_{2} \frac{\partial \varepsilon}{\partial x_{3}}=0$

Rewriting Eq. 11 and 12 in matricial form, and realizing that the trivial solution $\lambda=0$ can not satisfy the problem, it follows that the determinant of the system must be null. That condition is expressed as:

$\mu \frac{\partial \varepsilon}{\partial \mathrm{x}_{3}}-\varepsilon \frac{\partial \mu}{\partial \mathrm{x}_{3}}=0$

Outside the singular arc, the control variable $u$ is at one of its extreme values, denoted here as $u^{c}$. At the start of the integration, $u^{c}=0$. Hence, the DAE system presents index 1. The numerical integration continues until the solution trajectory intersects the singular trajectory. This intersection is detected when the left hand side of Eq. 13 vanishes. From that time on, the solution trajectory is coincident with the singular arc, and the constraint represented by Eq. 13 must be enforced. The resulting DAE system presents index 3 . When the maximum volume of the reactor is reached, no further addition of substrate is possible, and again $u$ is set as a constant value $\left(u^{c}=0\right)$. The DAE system presents index 1 again. The complete model, consequently, is a DAE of floating index type.

Equation 14 represents the weighted sum of all disjunctive equations, and the DAE system integrated consists of Eq. 5 to 7 and 14 , with state vector $y=\left[x_{1} x_{2} x_{3} u\right]^{T}$. The initial conditions at $\mathrm{t}=0$ and all parameters needed are reproduced at Table 1 .

$\eta(\mathrm{t})\left(\mathrm{u}-\mathrm{u}^{\mathrm{c}}\right)+(1-\eta(\mathrm{t})) \cdot\left(\mu \frac{\partial \varepsilon}{\partial \mathrm{x}_{3}}-\varepsilon \frac{\partial \mu}{\partial \mathrm{x}_{3}}\right)=0$ 
Table 1. Initial conditions and parameters used.

\begin{tabular}{|c|c|c|c|}
\hline $\mathrm{x}_{0}=1.0 \mathrm{~g} / \mathrm{L}$ & $\mathrm{K}_{1 \mathrm{p}}=16 \mathrm{~g} / \mathrm{L}$ & $\mathrm{u}_{\max }=10.0 \mathrm{~L} / \mathrm{h}$ & $\mu_{0}=0.408 \mathrm{~h}^{-1}$ \\
$\mathrm{~S}_{0}=150.0 \mathrm{~g} / \mathrm{L}$ & $\mathrm{K}_{2 \mathrm{p}}=71.5 \mathrm{~g} / \mathrm{L}$ & $\mathrm{u}_{\min }=0.0 \mathrm{~L} / \mathrm{h}$ & $\mathrm{g}_{0}=1.0 \mathrm{~h}^{-1}$ \\
$\mathrm{P}_{0}=10^{-5} \mathrm{~g} / \mathrm{L}$ & $\mathrm{K}_{1 \mathrm{~s}}=0.22 \mathrm{~g} / \mathrm{L}$ & $\mathrm{V}_{\max }=200.0 \mathrm{~L}$ & $\mathrm{c}=10 \mathrm{~g}$ \\
$\mathrm{~V}_{0}=10.0 \mathrm{~L}$ & $\mathrm{~K}_{2 \mathrm{~s}}=0.44 \mathrm{~g} / \mathrm{L}$ & $\mathrm{S}_{\mathrm{f}}=150 \mathrm{~g} / \mathrm{L}$ & $\mathrm{t}_{\mathrm{f}}=62 \mathrm{~h}$ \\
\hline
\end{tabular}

Figure 2 shows simulation results for the volume of the reactor and the substrate feed rate $u$. The control action is initially null, and presents a sudden variation at the beginning of the singular arc when the constraint (Eq. 13) becomes active. It can be noticed a small disturbance in the profile of this variable during the model switching interval, probably due to lack of precision of the integration code when the index is changing. The singular arc is followed until the maximum volume is reached. The control variable is again set to 0 . It must be emphasized that no reinitialization algorithm was used. The jump observed in the discontinuous variable $u$ was obtained automatically by the integration code.
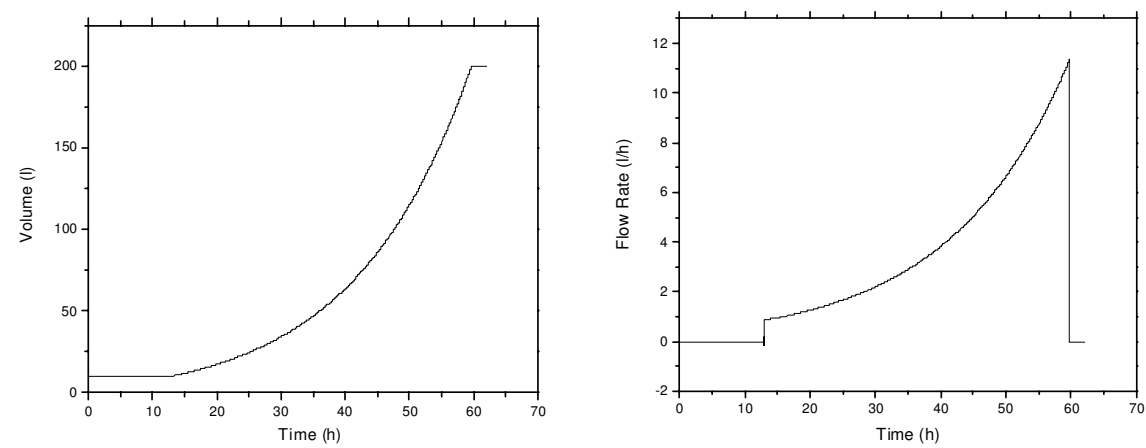

Figure 2 - Simulation results: reactor volume and substrate feed flowrate.

Figure 3 presents the simulation results for mass of cells and of product. It can be noticed that the oscillations in the substrate feed rate have no effect in the simulated results for the other variables, confirming the adequacy of the approach.
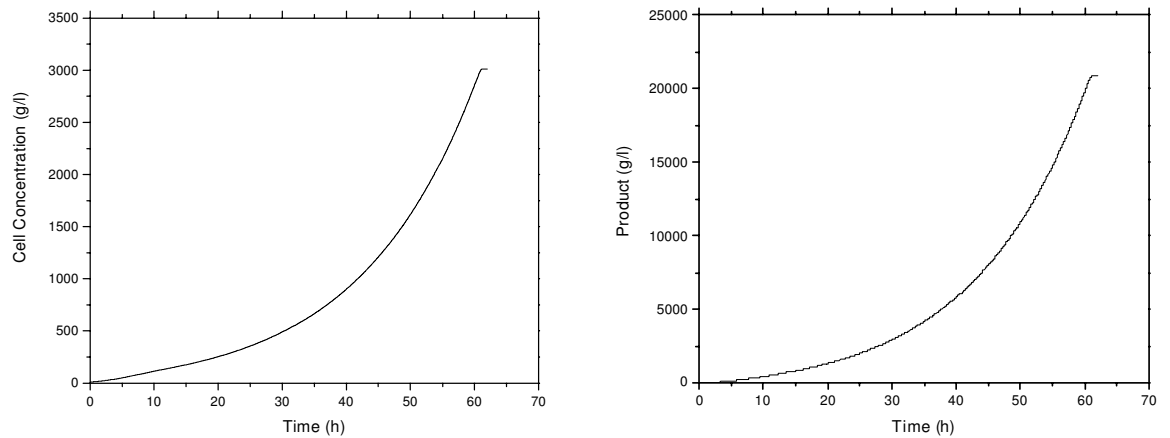

Figure 3 - Simulation results: biomass and ethanol concentration. 


\section{Conclusions}

In this paper a novel strategy for resolution of floating index DAE systems is presented. The smooth switching of models via a regularization function has been effective and adequate for all examples studied. The main advantages of the technique are:

- reduces algebraic manipulation for index reduction;

- no reinitialization strategy needed;

- ease of computational implementation;

- low computational effort.

The example presented in this contribution, despite simple, illustrates the application of the ideas discussed to a floating index DAE, demonstrating the robustness and simplicity of the approach. Additional examples have been studied by the authors and the results obtained have always been encouraging.

It should be pointed out that the methodology also applies for systems with model discontinuities which do not imply in index variation. The numerical simulation of simulated moving bed processes (inherently discontinuous) and fixed bed dryers (when the phenomena of inversion of flux can be observed) are typical examples.

The authors are presently investigating the application of the technique to optimal control systems with multivariable control, with emphasis on strategies for the detection of activation and deactivation of constraints.

\section{References}

ASCHER, U. M., PETZOLD, L., 1998, Computer Methods for Ordinary Differential Equations and Differential-Algebraic Equations, Classics in Applied Mathematics Series, SIAM.

FEEHERY, W. F., BARTON, P. I., 1996, "Dynamic Simulation and Optimization with Inequality Path Constraints", Computers and Chemical Engineering, v. 20, Suppl., pp.707-712.

FEEHERY, W. F., BARTON, P. I., 1998, "Dynamic Optimization With State Variable Path Constraints", Computers and Chemical Engineering, v. 22, 9, pp.1241-1256.

HONG, J., 1986, "Optimal Substrate Feeding Policy for a Fed Batch Fermentation with Substrate and Product Inhibition Kinetics", Biotechnology and Bioengineering, v. 28, pp. 1421-1431.

LIOEN, W. M., DE SWART, J. J. B., VAN DER VEEN, W. A., 1998, "PSIDE users guide", Report MAS-R9834, CWI, Amsterdam, Holanda. URL: http://www.cwi.nl/cwi/projects/PSIDE.

MAJER, C., MARQUARDT, W., GILLES, E. D., 1995, "Reinitialization of DAE's After Discontinuities", Computers and Chemical Engineering, v. 19, Suppl., pp.507-512.

PARK, T., BARTON, P. I., 1996, "State Event Location in Differential-Algebraic Models", ACM Transactions on Modeling and Computer Simulation, v. 6, nº 2, pp. 137-165.

PONTRYAGIN, L. S., BOLTYANSKII, V. G., GAMKRELIDZE, R. V., MISCHENSKO, Y.F., 1963, The Mathematical Theory of Optimal Processes, $1^{\text {st }}$ ed, Interscience, 1963.

VASSILIADIS, V. S., SARGENT, R. W. H., PANTELIDES, C. C., 1994a, "Solution of a Class of Multistage Dynamic Optimization Problems. 1. Problems without Path Constraints", Process Design and Control by Ind. Eng. Chem. Res., v. 33, pp. 2111-2122.

VASSILIADIS, V. S., SARGENT, R. W. H., PANTELIDES, C. C., 1994b, "Solution of a Class of Multistage Dynamic Optimization Problems 2. Problems with Path Constraints", Process Design and Control by Ind. Eng. Chem. Res., v. 33, pp. 2123-2133.

VIEIRA, R. C., BISCAIA Jr., E. C. ,2000, "Direct Methods for Consistent Inicialization of DAE Systems", Computers and Chemical Engineering, v. 25, pp. 1299-1311.

WU, B., WHITE, R. E., 2001, "An Initialization Subroutine for DAEs Solvers: DAEIS", Computers and Chemical Engineering, v. 25, pp. 301-311. 